\title{
MATHEMATICAL MODELS OF HYSTERESIS \\ (DYNAMIC PROBLEMS IN HYSTERESIS)
}

\section{FINAL REPORT}

The research undertaken under the grant No. DE-FG02-96ER14614 has been in complete compliance with the work statement of the grant. This research has further advanced the current state of the art in the areas of dynamic aspects of hysteresis and nonlinear large scale magnetization dynamics. The results of this research will find important engineering applications in the areas of magnetic data storage technology and the emerging technology of "spintronics".

Our research efforts have been focused on the following tasks:

- Study of fast (pulse) precessional switching of magnetization in magnetic materials.

- Analysis of critical fields and critical angles for precessional switching of magnetization.

- Development of inverse problem approach to the design of magnetic field pulses for precessional switching of magnetization.

- Study of magnetization dynamics induced by spin polarized current injection.

- Construction of complete stability diagrams for spin polarized current induced magnetization dynamics.

- Development of the averaging technique for the analysis of the slow time scale magnetization dynamics.

- Study of thermal effects on magnetization dynamics by using the theory of stochastic processes on graphs. 
Conceptually, the study of nonlinear magnetization dynamics has been carried out within the framework of Landau-Lifshitz and Landan-Lifshitz-Slonczewski equations. The analysis of ultra fast precessional switching of magnetization in nanostructures has been performed and new analytical tools for the calculation of critical pulse fields, critical angles and magnetic field pulse durations have been developed. A novel approach to the study of magnetization dynamics induced by the injection of spin polarized currents has been proposed and complete stability diagrams for this dynamics have been established. Novel stochastic differential equations on graphs for free magnetic energy have been derived and extensively used for the analysis of thermal effects.

The main results obtained in our research work on this grant have been presented at major international conferences such as INTERMAG and MMM (Magnetism and Magnetic Material) Conferences. These results have also been extensively published in such reputable journals as Physical Review Letters, Physical Review E, Journal of Applied Physics, IEEE Transactions on Magnetics, Physica B (Condensed Matter), Journal of Magnetism and Magnetic Materials, etc. These results are also presented in the scientific monograph of I.D. Mayergoyz entitled "Mathematical Models of Hysteresis and Their Applications", Academic Press-Elsevier (2003, pp. 1-472) and in the three-volume treatise “The Science of Hysteresis" Academic Press-Elsevier (2006, over 2100 pages) edited by G. Bertotti and I.D. Mayergoyz. The list of publications generated in the course of our work on the grant is attached.

Our scientific publications are frequently referenced. According to the data provided by the Institute for Scientific Information our publications were referenced in about 2000 scientific articles and the total number of citations exceeds 3000 . 
One PhD student Michai Dimian was supported by this grant and in 2005 he defended his thesis entitled "Nonlinear Spin Dynamics and Ultra-Fast Precessional Switching." After graduation, M. Dimian has obtained a prestigious postdoc position at Max Planck Institute, Germany, and in the Fall 2006 he will start his academic career as an Assistant Professor of Electrical Engineering at Howard University (Washington, DC).

\section{Publication List}

\section{a) Books}

1. I. Mayergoyz, "Mathematical Models of Hysteresis and Their Applications", AcademicPress-Elsevier, 2003.

2. G. Bertotti and I. Mayergoyz, (Editors), "The Science of Hysteresis", three volume treatise by Academic Press-Elsevier, 2006.

\section{b) Scientific Papers}

3. Bertotti, G., Mayergoyz, I.D., Serpico, C., D’Aquino, M., "Geometrical Analysis of Precessional Switching and Relaxation in Uniformly Magnetized Bodies," IEEE T. Magn. 39 (5): 2501-2503 Part 2, Sept. 2003.

4. Bertotti, G., Mayergoyz, I.D., Serpico,D., "Critical fields and Pulse Durations for Precessional Switching of thin Magnetic Films", IEEE T. Magn. 39(5): 2504-2506 Part 2 Sept. 2003.

5. Bertotti, G., Mayergoyz, I.D., Serpico, C., Dimian, M., "Comparison of Analytical Solutions of Landau-Lifshitz Equation for 'Damping' and 'Precessional' Switchings”, J. Appl. Phys. 93(10):6811-6813 Part 2 May 15, 2003.

6. Mayergoyz, I.D., Dimian, M., "Analysis of Spectral Noise Density of Hysteretic Systems Driven by Stochastic Processes”, J. Appl. Phys. 93(10):6826-6828 Part 2, May 15, 2003. 
7. Serpico, C., Mayergoyz, I.D., Bertotti, G., “Analyical Solutions of Landau-Lifshitz Equation for Precessional Switching”, J. Appl. Phys. 93(10):6909-6911 Part 2, May $5,2003$.

8. Magni, A., Bertotti, G., Mayergoyz, I.D., Serpico, C., "Landau-Lifschitz-Gilbert Dynamics and Eddy Current Effects in Metallic Thin Films”, J. Magn. Magn. Mater 254:210-212 Sp. Iss. Si January 2003.

9. Dimian, M., Mayergoyz, I.D., "Spectral density analysis of nonlinear hysteretic systems”, Phys. Review E 70(4): Art. No. 046124 Part 2, October 2004.

10. Dimian, M., Mayergoyz, I.D., "Spectral noise density of the Preisach model", IEEE Trans. On Magnetics 40(4):2134-2136 Part 2, July 2004.

11. Serpico, C., d'Aquino, M., Bertotti, G., Mayergoyz, I.D., "Analysis of quasiperiodic Landau-Lifshitz-Gilbert dynamics", Journal of Magnetism and Magnetic Materials, 272-76:734-735 Part 1, Sp Iss. SI May 2004.

12. Bertotti, G., Mayergoyz, I.D., Serpico, C., "Averaging technique for the analysis of magnetization relaxations", Journal of Applied Physics 95(11):6598-6600 Part 2, June 1, 2004.

13. Mayergoyz, M., Dimian, M., Bertotti, G., Serpico, C., "Inverse problem approach to the design of magnetic field pulses for precessional switching", Journal of Applied Physics 95(11):7004-7006 Part 2, June 1, 2004.

14. Bertotti, G., Bonin, R., Mayergoyz, I.D., Serpico, C., "Generalized magnetostatic modes around large magnetization motions", Journal of Applied Physics 95(11): 7046-7048, Part 2, June 1, 2004. 
15. Serpico, C., d'Aquino, M., Bertotti, G., Mayergoyz, I.D., "Quasiperiodic magnetization dynamics in uniformly magnetized particles and films", Journal of Applied Physics 95(11): 7052-7054 Part 2, June 1, 2004.

16. Brauer, J.R., Mayergoyz, I.D., "Finite-element computation of nonlinear magnetic diffusion and its effects when coupled to electrical, mechanical, and hydraulic systems", IEEE Transactions on Magnetics 40(2):537-540, Part 2, March 2004.

17. Bertotti, G., Mayergoyz, I.D., Serpico, C., “Analytical solutions of Landau-Lifshitz equation for precessional dynamics", Physica B-Condensed Matter 343(1-4):325330, January 1, 2004.

18. Bertotti, G., Serpico, C., Mayergoyz, I.D., Magni, A., d'Aquino, M., Bonin, R., "Magnetization switching and microwave oscillations in nanomagnets driven by spin-polarized currents", Physical Review Letters, 94(12): Art. No. 1272006, April $1,2005$.

19. Bertotti, G., Bonin, R., Magni, A., Mayergoyz, I.D., Serpico, C., "Analytical description of magnetization relaxation to equilibrium", Journal of Applied Physics 97(10): Art. No. 0E315 Part 2, May 15, 2005.

20. d'Aquino, M., Serpico, C., Miano, G., Mayergoyz, I.D., Bertotti, G., "Numerical integration of Landau-Lifshitz-Gilbert equation based on the mid-point rule, Journal of Applied Physics 97(10): Art. No. 10E319 Part 2, May 15, 2005.

21. Dimian, M., Mayergoyz, I.D., "Influence of surface anisotropy on the magnetization precessional switching in nanoparticles", Journal of Applied Physics 97(10): Art. No. 10J302 Part 3, May 15, 2005. 
22. Mayergoyz, I.D., Dimian, M., Bertotti, G., Serpico, C., "Inverse problem approach to precessional switching in perpendicular media", Journal of Applied Physics 97 (10): Art. No. 10A703 Part 2, May 15, 2005.

23. Mayergoyz, I.D., Dimian, M., Bertotti, G., Serpico, C., "Critical fields and pulse durations for precessional switching of perpendicular media", Journal of Applied Physics 97(10): Art. No. 10E509 Part 2, May 15, 2005.

24. Bertotti, G., Serpico, C., Mayergoyz, I.D., Bonin, R., "Magnetization selfoscillations induced by spin-polarized currents", IEEE Transactions on Magnetics 41(10):2574-2576, October 2005.

25. Serpico, C., Bertotti, G., d'Aquino, M., Mayergoyz, I.D., “Transient dynamics leading to self-oscillations in nanomagnets driven by spin-polarized currents", IEEE Transactions on Magnetics 41(10):3100-3102, October 2005.

26. Serpico, C., d'Aquino, M., Bertotti, G., Mayergoyz, I.D., "Analytical approach to current-driven self-oscillations in Landau-Lifshitz-Gilbert dynamics", Journal of Magnetism and Magnetic Materials 290:502-505 Part 1, Sp. Iss. SI, April 2005.

27. Bertotti, G., Magni, A., Bonin, R., Serpico, C., Mayergoyz, I.D., "Bifurcation analysis of magnetization dynamics driven by spin transfer", Journal of Magnetism and Magnetic Materials 290:522-525 Part 1, Sp. Iss. SI, April 2005.

28. Bertotti, G., Bonin, R., Magni, A., Mayergoyz, I.D., Serpico, C., "Energy equation for the analysis of magnetization relaxation to equilibrium", Journal of Magnetism and Magnetic Materials 286: 282-285, Sp. Iss. SI, February 1, 2005.

29. Mayergoyz, I.D., Dimian, M., "Stochastic aspects of hysteresis", Journal of Physics: Conference Series 22, 139-147, 2005. 
30. d'Aquino, M., Serpico, C., Coppola, G., Mayergoyz, I.D., Bertotti, G., "Midpoint numerical technique for stochastic Landau-Lifshitz-Gilbert dynamics", Journal of Applied Physics 99 (8): Art. No. 08B905, April 15, 2006.

31. Bertotti, G., Mayergoyz, I.D., Serpico, C., "Analysis of random Landau-Lifshitz dynamics by using stochastic processes on graphs", Journal of Applied Physics 99 (8): Art. No. 08F301, April 15, 2006.

32. Dimian, M., Mayergoyz, I.D., Bertotti, G., Serpico, C., "Multiscale analysis of magnetization dynamics driven by external fields", Journal of Applied Physics 99 (8): Art. No. 08G104, April 15, 2006.

33. Serpico, C., Bertotti, G., Mayergoyz, I.D., Bonin, R., "Thermal stability in spintorque-driven magnetization dynamics", Journal of Applied Physics 99 (8): Art. No. 08G505, April 15, 2006.

34. Bonin, R., Bertotti, G., Mayergoyz, I.D., Serpico, C., "Spin-torque-driven magnetization dynamics in nanomagnets subject to magnetic fields perpendicular to the sample plane", Journal of Applied Physics 99 (6): Art. No. 08G508, April 15, 2006.

35. Belbas, S.A., Mayergoyz, I.D., "Hadamard-like derivatives in Preisach modeling and control", Physica B-Condensed Matter 372 (1-2): 87-90 February 1, 2006. 